\title{
Student ability to distinguish between superposition states and mixed states in quantum mechanics
}

\author{
Gina Passante, Paul J. Emigh, and Peter S. Shaffer \\ Department of Physics, University of Washington, Seattle, Washington 98195, USA
}

(Received 1 June 2015; published 25 November 2015)

\begin{abstract}
Superposition gives rise to the probabilistic nature of quantum mechanics and is therefore one of the concepts at the heart of quantum mechanics. Although we have found that many students can successfully use the idea of superposition to calculate the probabilities of different measurement outcomes, they are often unable to identify the experimental implications of a superposition state. In particular, they fail to recognize how a superposition state and a mixed state (sometimes called a "lack of knowledge" state) can produce different experimental results. We present data that suggest that superposition in quantum mechanics is a difficult concept for students enrolled in sophomore-, junior-, and graduate-level quantum mechanics courses. We illustrate how an interactive lecture tutorial can improve student understanding of quantum mechanical superposition. A longitudinal study suggests that the impact persists after an additional quarter of quantum mechanics instruction that does not specifically address these ideas.
\end{abstract}

\section{INTRODUCTION}

Superposition is a core concept in quantum mechanics. Particles can exist in superposition states for which multiple measurement values are possible for a given observable. Although a single value is observed upon measurement, the particle cannot be treated as having that value prior to the measurement. Different interpretations of quantum mechanics may describe such superposition states in different ways, but regardless of the details of the interpretation, such states play a crucial role in the theory of quantum mechanics.

Superposition is often one of the first quantum mechanical concepts taught. For example, it is central to the electron double-slit experiment, which is often used to motivate why electrons cannot be treated as classical particles [1]. Recently, there has been a tendency to introduce quantum mechanical ideas, such as superposition, earlier and earlier in physics instruction. The ideas are sometimes presented as early as introductory university physics or high school [2-4]. However, at these levels, the mathematical formalism of quantum mechanics is typically not taught because students lack the prerequisite mathematics. Therefore, the focus is often on conceptual understanding (see, for example, Refs. [5-7]). There have been several researchbased reforms that attempt to improve this type of instruction [5,6,8-12]. Although students have the prerequisite knowledge for learning superposition at this level, there has

Published by the American Physical Society under the terms of the Creative Commons Attribution 3.0 License. Further distribution of this work must maintain attribution to the author(s) and the published article's title, journal citation, and DOI. been disagreement among faculty regarding its importance in a modern physics course [13].

In this paper, we focus on student understanding of a particular aspect of superposition in quantum mechanics: the ability to distinguish between a superposition state, which is inherently quantum mechanical, and a mixed state, which can be described classically as lack of knowledge about the system [14]. We describe the results of an investigation into whether or not students at all levels of instruction can distinguish between these two types of states. We also discuss a lecture tutorial suitable for the sophomore level that we are developing to address this idea, and report on its impact in a longitudinal study.

Previous research has shown that students struggle with the idea of superposition in quantum mechanics in several ways [15-17]. For example, in the context of the SternGerlach experiment, Zhu and Singh [16] asked students to determine a strategy for distinguishing between an ensemble of superposition particles and an ensemble containing a mixture of up and down spin states. They found that few students were able to do this successfully. In this paper we focus on a prerequisite question: whether or not students recognize that the two types of states are, in fact, different, and the reasoning they use to justify their answers.

This paper has three main components. The context for this investigation is outlined in Sec. II. We then (i) present results from questions that were used to probe student ability to distinguish superposition states from mixed states throughout undergraduate and graduate quantum mechanics courses (Sec. III); (ii) discuss an intervention designed to improve student understand of this idea at the sophomore level (Sec. IV); and (iii) document the shortand long-term effects of the intervention (Secs. VA and $\mathrm{V} B$, respectively). 


\section{CONTEXT FOR INVESTIGATION}

This investigation has taken place in three courses on quantum mechanics at the University of Washington (UW): a sophomore-, junior-, and graduate-level course.

The sophomore course is required of all physics majors. It uses a spins-first approach and covers the first five chapters in David McIntyre's Quantum Mechanics textbook [18]. It is the only required quantum mechanics course for students in the applied physics degree track. (The comprehensive, teacher prep, and biological tracks require at least one-quarter of junior-level quantum mechanics.) The sophomore course is intended to provide an accessible introduction to quantum mechanics that does not rely on the full mathematical treatment typically covered in a junior-level course. This is necessary since students have not yet completed the prerequisite mathematics courses. The course also attempts to prepare students for future courses on quantum mechanics. During the investigation, the lecturers used interactive techniques such as online prelecture assignments to prepare students for lecture, clickers, and think-pair-share activities. Interactive lecture tutorials (i.e., tutorials adapted for use in a lecture setting) were also used [19]. The data from this course were collected through the use of clicker questions for which students received participation credit.

The junior-level course is the first of a two-quarter sequence that covers the entire Introduction to Quantum Mechanics textbook by David Griffiths [20]. During this investigation the course had two primary components: lectures and tutorials. The lectures were taught in a lecture hall to 50-90 students. Clickers were not used, although notfor-credit quizzes were administered several times a quarter to promote student engagement. Each student was also enrolled in a tutorial section consisting of no more than 30 students. In these sections students worked through Tutorials in Physics: Quantum Mechanics, materials that are being developed by our group. These tutorials have been developed to address common conceptual difficulties that we have identified and are in the same format as Tutorials in Introductory Physics [21]. Data were collected using weekly online questionnaires given after relevant lecture instruction but before tutorial. Students received credit for completing them but their answers were not graded.

The graduate courses are the first two courses in a threequarter sequence on quantum mechanics taken by students in their first year of graduate school. They were taught in a traditional lecture format. Approximately 30 students were enrolled. We gathered data in the form of written responses to a pre-course survey and a midterm exam.

\section{INVESTIGATION OF STUDENT UNDERSTANDING}

This investigation has focused on the ability of students to distinguish between a quantum mechanical superposition state (also known as a pure superposition state) and a mixed state. Student ability to distinguish them has been examined in the three courses at various levels described in the previous section.

The primary question used has been asked in three different contexts: spin-1/2 particles (question 1 , shown in Fig. 1), infinite square well energy eigenstates (question 2, shown in Fig. 2), and hydrogen atom energy eigenstates (question 3, shown in Fig. 3). All three questions describe two collections (or ensembles) of identically prepared particles. One collection contains pure states prepared in an equal superposition of $S_{z}$ eigenstates (for question 1) or of two energy eigenstates (for questions 2 and 3). The other collection has half of the particles in one state and the other half of the particles in the other state. The questions ask students to determine which statement describes the possible experimental properties of these collections. The correct statement, option $C$, indicates that there is an experimental difference between the two collections. For example, an experiment that would distinguish the collection in question 1 would be to measure the spin of the particles in the $x$ basis. Option $A$ states that the two collections are the same and no experiment can distinguish between them; option $B$ says that although the collections are different, there is no experiment that can tell them apart.

In all three classes, the question was administered after relevant lecture instruction. (In the case of the graduate course, students had seen the material in previous undergraduate courses.) Below we describe student performance.

\section{A. Results from the sophomore course}

In the sophomore course, question 1 (Fig. 1) was administered as a clicker question during lecture. Instruction had covered spin and the Stern-Gerlach apparatus. The lecturer had shown students a PhET simulation on the Stern-Gerlach apparatus [22]. In addition, students had worked through an interactive lecture tutorial on spin

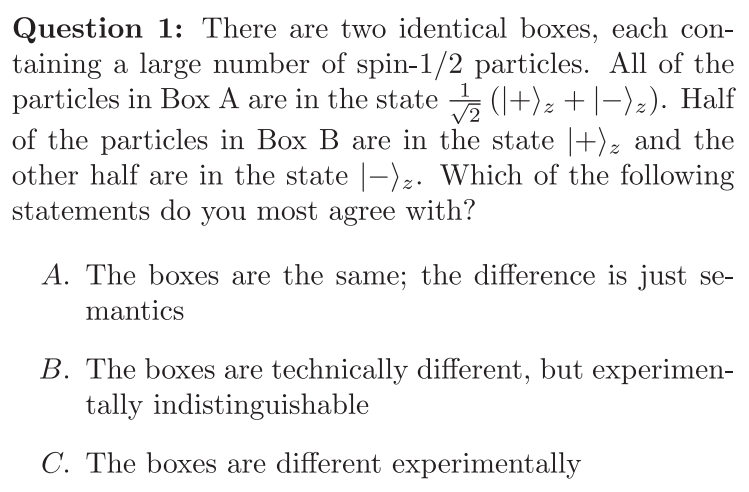

Question 1: There are two identical boxes, each containing a large number of spin- $1 / 2$ particles. All of the particles in Box A are in the state $\frac{1}{\sqrt{2}}\left(|+\rangle_{z}+|-\rangle_{z}\right)$. Half of the particles in Box B are in the state $|+\rangle_{z}$ and the other half are in the state $|-\rangle_{z}$. Which of the following statements do you most agree with? $A$. The boxes are the same; the difference is just se-
mantics
$B$. The boxes are technically different, but experimen- tally indistinguishable
C. The boxes are different experimentally

FIG. 1. Question on superposition in the context of spin. This question has been given in this form to sophomore students. A variant has also been given to students at the beginning of graduate-level instruction. 
Question 2: Imagine two ensembles of infinite square wells. Ensemble A has all of the particles in the state $\frac{1}{\sqrt{2}}\left(\psi_{1}+\psi_{2}\right)$. Ensemble B has half of the particles in $\psi_{1}$ and half in $\psi_{2}$. Which statement best describes the differences or similarities between the two ensembles?
$A$. The boxes are the same; the difference is just se- mantics
$B$. The boxes are technically different, but experimen- tally indistinguishable
C. The boxes are different experimentally

FIG. 2. Superposition question in the context of the infinite square well. Students were informed that $\psi_{n}$ is the $n$th energy eigenstate of the infinite square well. Note that the answer options are identical to those asked in question 1 in Fig. 1. This question was given to students at the sophomore level.

[23]. The question was asked after students had all the tools necessary to answer (i.e., the ability to write a spin state in several different bases and to determine the probability of a measurement outcome).

The first row in Table I shows the percentage of students who chose each answer option on question 1 [24]. The data are aggregated across four classes $(N=277)$, all with very similar results (within 10\%). Overall, approximately $43 \%$ of the students answered correctly, while the other $57 \%$ answered that the two boxes cannot be distinguished (either because they are inherently the same, or because there is no experiment that can distinguish between them).

After the students had finished the clicker question, the lecturer went through a detailed solution and discussed two experiments that could be performed on the boxes. The first involves the use of a Stern-Gerlach device oriented in the $z$ direction. This experiment produces identical results for the two boxes. The second experiment uses a Stern-Gerlach device oriented in the $x$ direction. In this case, the results for the two boxes differ: each particle in box $A$ is measured to be spin-up, while approximately half of the particles in box $B$ are measured to be spin-up and the other half spin-down.

In order to probe the extent to which students had understood the relevant concepts as a result of this additional instruction, question 2 (Fig. 2) was administered as a clicker question near the end of the quarter in two courses $(N=58)$. The difference between superposition and mixed

TABLE I. Percentage of sophomore-level students who chose each answer option for questions 1 and 2 . The number of students selecting each option is given in parentheses.

\begin{tabular}{lcll}
\hline \hline & $A$ & \multicolumn{1}{c}{$B$} & \multicolumn{1}{c}{$C$} \\
\hline Question 1 $(N=277)$ & $14 \%(40)$ & $42 \%(117)$ & $43 \%(118)$ \\
Question 2 $(N=58)$ & $7 \%(4)$ & $47 \%(27)$ & $47 \%(27)$ \\
\hline \hline
\end{tabular}

TABLE II. Response patterns for students who answered both question 1 and question $2(N=47)$.

\begin{tabular}{lc}
\hline \hline Question $1 \rightarrow$ Question 2 & Percentage (Number) \\
\hline Incorrect $\rightarrow$ Incorrect & $38 \%(18)$ \\
Incorrect $\rightarrow$ Correct & $30 \%(14)$ \\
Correct $\rightarrow$ Incorrect & $13 \%(6)$ \\
Correct $\rightarrow$ Correct & $19 \%(9)$ \\
\hline \hline
\end{tabular}

states had not been directly addressed or tested since the first clicker question had been given.

Student performance was very similar on both questions; slightly less than half correctly answered both questions. The results are shown in Table I. Of the students who answered both questions, approximately half were not consistent in their answers. (See Table II. Note that some students who were incorrect on both questions were not consistent in their answers and flipped between options $A$ and $B$.) These findings suggest that students had not developed a functional understanding of the difference between the two ensembles. Some may not have understood the material at any point or may have forgotten. Others may have been unable to transfer their knowledge to the new context. In either case, the results demonstrate how difficult quantum mechanical superposition can be for students.

\section{B. Results from the junior course}

At the end of the first quarter of the junior-level sequence, students were given question 3, shown in Fig. 3. The answer options were worded somewhat differently than on the other versions (shown in Figs. 1 and 2), but test the same ideas. Performance by the juniors was

Question 3: Consider the following collections:

Collection 1: A large number of particles, each in an equal superposition of the ground state and the first excited state of the Hydrogen atom, $\psi=\frac{1}{\sqrt{2}}\left(\psi_{100}+\psi_{200}\right)$.

Collection 2: A large number of particles, half of which are in the ground state of the Hydrogen atom and half of which are in the first excited state.

Is there an experiment that can distinguish between these two collections?

a) No, there is no such experiment because the two collections are identical.

b) No, there is no such experiment even though the collections are different.

c) Yes, there is such an experiment because the collections are different.

FIG. 3. Superposition question given at the end of the first quarter of junior-level quantum mechanics instruction. 
similar to that of students in the sophomore course: $37 \%$ (11 out of 30) answered correctly. (The data reported in the Table include only those students in the junior course who had not seen a version of this question in the sophomore course. The results from the other students who had worked through an instructional intervention are discussed in Sec. V B 2.)

\section{Results from the graduate course}

A variation of question 1 (Fig. 1) was also given to students in the first week of a graduate-level quantum mechanics course. The version used differed only slightly from that shown in the figure in that it specified that each box had 100 particles in it (as opposed to a large number). Students were also asked to explain their reasoning.

The results are shown in Table III. A total of 31 students responded. Nearly half selected the incorrect options $A$ and $B$ (16\% and $29 \%$, respectively). About $45 \%$ gave correct answers, however, their reasoning was nearly evenly split between two categories (labeled $C 1$ and $C 2$ in the table). About $19 \%$ gave entirely correct reasoning, stating that a measurement of spin in the $x$ direction would differentiate between the two categories of particles. (These students are labeled $C 2$ in the table.) The remaining $26 \%$ of the students reasoned on the basis of the probabilistic nature of the measurement of spin. They state that since the particles in collection $A$ are in the state $\frac{1}{\sqrt{2}}(|\uparrow\rangle+|\downarrow\rangle)$, then a measurement of spin in the $z$ direction, yields a $50 \%$ probability of measuring spin-up. Thus, it is possible that only 49 particles might be found to be spin-up. This is in contrast to collection $B$, which must have a perfect 50/50 split. This reasoning is labeled as $C 1$ in the Table. Although this line of reasoning is correct, it does not focus on the fundamental difference between the two ensembles, but rather on the probabilistic nature of the measurements. These results indicate that even graduate students struggle with recognizing the difference between pure superposition states and mixed states.

In the graduate course, a question similar to the one shown in Fig. 1 was asked on the midterm exam after density operators were covered in lecture as a method for writing mixed states. It is at this point in the course that the mathematical difference between a pure and a mixed quantum state is taught. Unlike when this question was

TABLE III. Responses to a variant of question 1 from the beginning of the first quarter of a graduate course on quantum mechanics. The correct answer $(C)$ has been divided into two categories based on the reasoning students provided: category $C 2$ is considered correct with correct reasoning. (This distinction is described in more detail in the text.)

\begin{tabular}{ccccc}
\hline \hline & $A$ & $B$ & $C 1$ & $C 2$ \\
\hline Question 1 $(N=31)$ & $16 \%(5)$ & $29 \%(9)$ & $26 \%(8)$ & $19 \%(6)$ \\
\hline \hline
\end{tabular}

asked at the beginning of the course, this time the question did not specify the number of particles in each ensemble but only indicated that it was a large number. On the exam, 93\% (25 of 27) of the students provided both a correct answer and a correct explanation. They did so by either indicating that a measurement in the $x$ direction would differentiate between the two ensembles (20/27) and/or they tested for purity by evaluating the trace of the density operator squared $(9 / 27)$. When the trace is equal to one, the state is pure, while if the trace is less than one, the state is mixed.

These results suggest that the density operator formalism is a useful tool for students to use when answering this type of question. The question remains whether or not this mathematical formalism is necessary in order for students to be able to distinguish pure superposition states and mixed states. The difference between these two types of states is not only mathematical, but has qualitative importance due to the fact that there is an experimental difference between them.

\section{LECTURE TUTORIAL ON SUPERPOSITION}

We wanted to design instructional materials to help sophomore-level students recognize and understand the difference between pure superposition states and mixed states qualitatively, without the advanced mathematical formalism. Constrained by the lack of small group sections in the sophomore course, we created an interactive lecture tutorial. It has been modeled after Tutorials in Introductory Physics [21], which have been demonstrated to be effective at improving student understanding at the introductory level $[25,26]$. We were inspired by others who have documented their success using and adapting the introductory tutorials for large lecture halls [19].

The interactive lecture tutorial that we developed to help students work through the ideas of superposition states and mixed states is entitled "Superposition in Quantum Mechanics" [27]. It was used near the end of the sophomore course, after students had learned about the solutions to the Schrödinger equation for the infinite square well. The interactive lecture tutorial has students think about the different types of experiments that could be performed in an attempt to distinguish between the two collections from question 2 (Fig. 2).

When this interactive lecture tutorial has been used, the class periods began with a clicker question on superposition (question 2 in Fig. 2). Students were generally not given the opportunity to see the distribution of answers immediately after answering the question. Rather, they worked through the tutorial in small groups where they were seated. The instructor and several teaching assistants (TAs) were available to help students. There was approximately one TA for every 15-40 students (the number varied depending on the specific quarter). Students were engaged with the material and worked with their neighbors; 
groups would call a TA for assistance when they had difficulty answering a particular question. At various times during the class, the instructor would reconvene the students to discuss and summarize the findings.

\section{IMPACT OF LECTURE TUTORIAL}

The impact of the interactive lecture tutorial has been tested in two ways. First, we probed student understanding immediately after it was used in the sophomore course. We also tested retention twice, once at the start of the juniorlevel course and once at the end of the junior course.

\section{A. Immediate impact of lecture tutorial}

In one of the classes in which the lecture tutorial was used, question 2 was given as both a pretest and as a posttest immediately after the tutorial. (Recall that students were not given the opportunity to see the correct answer or the distribution of responses after the first administration of the question.) The results of both administrations of the question for this class are shown in Table IV.

The percentage of students who chose the incorrect option $B$ (that the ensembles are technically different, but experimentally indistinguishable) dropped from $50 \%$ to $9 \%$, and the percentage who chose option $C$ (the ensembles are different experimentally) increased from $47 \%$ to $91 \%$. The tutorial thus seemed to be successful, at least in the short term, in guiding students through the difference between a mixed state and an ensemble of states.

\section{B. Prolonged impact of the lecture tutorial}

In order to determine whether or not the tutorial had a lasting effect on student understanding, we followed sophomore students to the junior-level quantum mechanics course offered in the fall quarter. Students fell into one of three different categories:

(i) [No tutorial] Students who had taken the prerequisite sophomore quantum mechanics without the interactive lecture tutorial. $(N=51)$

(ii) [With tutorial-Winter] Students who had taken the prerequisite sophomore quantum mechanics course during the prior winter quarter with the interactive lecture tutorial. Six months had elapsed since they had completed the sophomore course. $(N=48)$

(iii) [With tutorial-Summer] Students who had taken the prerequisite sophomore quantum mechanics course during the prior summer quarter with the

TABLE IV. Responses to question 2 (Fig. 2) given immediately before and after the tutorial.

\begin{tabular}{lccc}
\hline \hline & $A$ & $B$ & $C$ \\
\hline Before tutorial $(N=32)$ & $3 \%(1)$ & $50 \%(16)$ & $47 \%(15)$ \\
After tutorial $(N=33)$ & $0 \%(0)$ & $9 \%(3)$ & $91 \%(30)$ \\
\hline \hline
\end{tabular}

interactive lecture tutorial. One month had elapsed since they had completed the sophomore course. $(N=32)$

Note that the primary difference between the $\beta$ and $\gamma$ categories is the amount of time between the sophomore and junior courses. Students in categories $\beta$ and $\gamma$ took the sophomore-level course during the winter and summer, respectively, and then took the junior-level course in the following autumn [28].

\section{Assessment at the start of the junior quarter}

To test the long-term effects of the tutorial, we asked students in all three categories a new question (Fig. 4) that did not share any surface features with those questions in the sophomore course (questions 1 and 2). It was given as part of a weekly online pretest that students received credit for completing but was not graded.

The question presented a quantum mechanical state that was written as a superposition and asked students to consider four statements about the superposition state. For each statement, students were asked whether they "completely agree," "partially agree," or "completely disagree." Each statement was on a different page of the online quiz and students could not backtrack to revise their previous answers. All four of the statements are false, so the correct answer for each is completely disagree. The percentage of the students who answered correctly is shown in Fig. 5.

Student explanations were used to classify the partially agree responses as either agreeing or disagreeing with each statement. An example of a response in which a student partially agreed with statement 1 ("the particle is in an

$$
\begin{aligned}
& \text { Consider the superposition state: } \psi=\frac{1}{\sqrt{3}} \psi_{1}+\sqrt{\frac{2}{3}} \psi_{2} \text {. } \\
& \text { For each of the statements below, indicate whether you } \\
& \text { agree, partially agree, or disagree and explain your rea- } \\
& \text { soning. } \\
& \text { S1: The particle is in an unknown state. } \\
& \text { S2: The state is either } \psi_{1} \text { or } \psi_{2} \text {, but we don't know } \\
& \text { which until after we measure. } \\
& \text { S3: The particle can be thought of as coming from a } \\
& \text { procedure that produces } \psi_{1} \text { one-third of the time } \\
& \text { and } \psi_{2} \text { two-thirds of the time. } \\
& \text { S4: If I measure the particle to be in } \psi_{2} \text {, then I know } \\
& \text { that immediately before the measurement, it was } \\
& \text { in } \psi_{2} \text {. }
\end{aligned}
$$

FIG. 4. Students were asked to agree, partially agree, or disagree with these four statements. Each statement appeared on a separate page of the online quiz and no backtracking was allowed. This question was given at the beginning of a juniorlevel quantum mechanics course. 


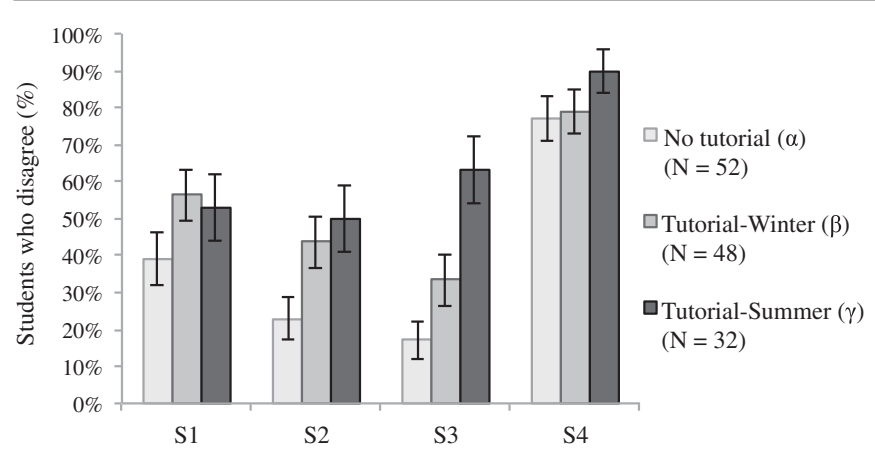

FIG. 5. The percentage of students by category $(\alpha, \beta$, or $\gamma)$, who answered each statement correctly. Error bars indicate the standard error on a proportion $[\sqrt{p(1-p) / N}]$.

unknown state") is given below. This response was treated as agreeing with the statement (and therefore is incorrect).

"The particle is either in the $\psi_{1}$ state or the $\psi_{2}$ state, which we can't tell, but it narrows it down from being in any possible state." [statement 1]

Some students who selected partially agree explained that they did so because the energy eigenstates were not defined (as we did not specify the potential). These students were counted as correct (disagreeing with the statement) if they indicated that they only needed information about the eigenstates (or the potential) in order to answer.

Both populations of students who had worked through the tutorial in their sophomore course were more likely to give a correct answer for each statement than the students who did not work through the tutorial. One-tailed $t$ tests were used to evaluate whether or not the performance of the students in category $\beta$ or $\gamma$ was different from the performance of students in category $\alpha$. Two-tailed $t$ tests were used for comparisons between categories $\beta$ and $\gamma$. The difference between the performance of both groups of students who did have the tutorial ( $\beta$ and $\gamma$ students) and those who did not have the tutorial $(\alpha)$ is significant (with $p<0.05$ ) on statements 1,2 , and 3 . (The only nonsignificant result is the difference between the $\alpha$ and $\gamma$ proportions on statement 1.) In addition, for most of the questions the difference between the students who had the tutorial in the summer and winter ( $\beta$ and $\gamma$ students) was not statistically significant; the exception is statement 3 $(t=2.16 ; d f=35.7 ; p=0.038)$.

Student explanations provide some insight into their thinking. Consider the answer given in response to statement 1 ("the particle is in an unknown state"):

"It is not known exactly which state the particle is in, but it is either in $\psi_{1}$ or $\psi_{2}$. " [statement 1]

It is possible that this student believes that a particle cannot be in a superposition state, but rather that the notation simply indicates the probability that the particle is in one of the options (in this case, $\psi_{1}$ or $\psi_{2}$ ). Another possibility is that the student is unclear about the meaning of the word "state," and thinks the term does not describe superpositions of energy eigenstates, but only the energy eigenstates themselves. This confusion has been seen in response to other questions.

Students who incorrectly agree with statement 2 seem to believe that the measurement will disturb the state, but that it is still in one state or the other before measurement.

"We would know which one after we measure, but until then it could be in either state. It's more likely to be in one than the other, but there [is] no way of knowing before measuring." [statement 2]

The third statement is where we found the most discrepancy between the summer and winter students. This statement is the most different from what students would have learned in lecture. It is meant to evoke the idea of a lack of knowledge of the state, by implying that the particle is in one or the other of the two eigenstates, but we simply do not know which. Students who answered correctly (disagreed with statement 3 ) were able to identify that the procedure would produce a mixed state and not a superposition.

"That would be a mixture, not a superposition like the above state is." [statement 3]

Students who answered incorrectly (agreed with the statement) tended to explain that correct probability values given in the statement were calculated correctly.

The fourth statement seemed to be the easiest for students. Approximately $80 \%$ of all students answered correctly. Many correctly reasoned that there is no way to know which state the particle is in until after the measurement. However, this type of explanation was given by students who both agreed and disagreed with the statement, indicating that this statement does not seem to be effective at distinguishing different levels of student understanding.

The results from all four statements indicate that many students struggle with the difference between superposition states and mixed states. One might expect that students who disagree with one of the statements would disagree with them all. However, we find this is not the case; Fig. 6 shows the number of statements disagreed with for each category of students. The average number of statements disagreed with is $1.66,2.33$, and 2.66 for students in the $\alpha, \beta$, and $\gamma$ categories, respectively. The difference between students who did not take the tutorial and either group who did take the tutorial is statistically significant $(P<0.01$ when comparing $\alpha$ and $\beta$, and $P<0.001$ when comparing $\alpha$ to $\gamma$ ). This shows that the difference between a superposition state and a mixed state is nuanced. Answering a 


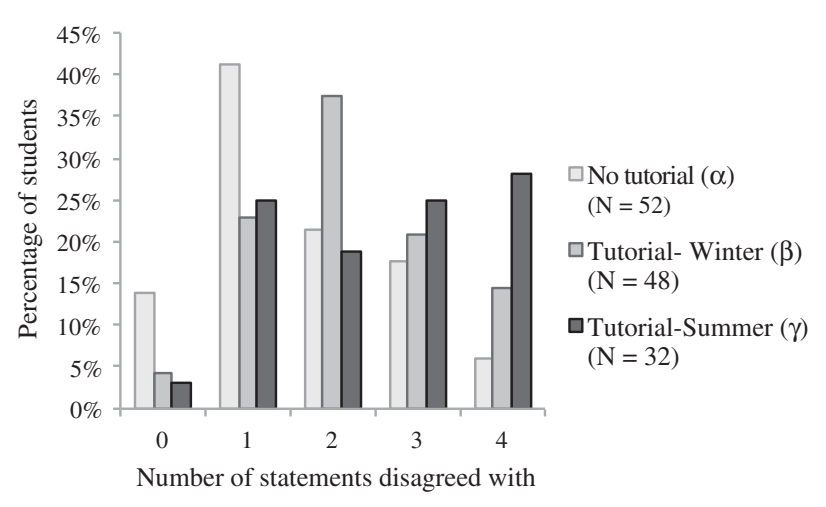

FIG. 6. The number of statements that students disagreed with. Only students who answered all four of the questions were included in these data.

TABLE V. Responses to question 3 (Fig. 3) administered at the end of a quarter of junior-level quantum mechanics instruction.

\begin{tabular}{lclc}
\hline \hline & $A$ & \multicolumn{1}{c}{$B$} & $C$ \\
\hline No Tutorial $\alpha(N=30)$ & $37 \%(11)$ & $27 \%(8)$ & $37 \%(11)$ \\
Tutorial-winter $\beta(N=62)$ & $8 \%(5)$ & $32 \%(20)$ & $60 \%(37)$ \\
Tutorial-summer $\gamma(N=17)$ & $6 \%(1)$ & $24 \%(4)$ & $71 \%(12)$ \\
\hline \hline
\end{tabular}

single question correctly does not necessarily indicate a complete understanding.

\section{End of the junior quarter}

We also tested student understanding of superposition states and mixed states at the end of the first quarter of the junior-level course. Question 3 (Fig. 3), described in Sec. III B was given online and students were given participation credit for answering. As on the previous questions, the correct answer is that there is an experiment that can distinguish between the two collections (option $C$ ). Measurements of the energy would not be sufficient, but measurements of position, for example, would provide different results for the two ensembles.

Table V shows the results for the three populations. Students who did not have the tutorial (category $\alpha$ ) had seen this type of question once before. However, the distribution of their answer choices is very similar to what we find when students are asked this question for the first time (see Table I). This supports the results from Sec. III in which we found that additional instruction in quantum mechanics does not necessarily improve performance on this type of question. The results for population $\alpha$ were also reported in Sec. III B. We found that $60 \%$ and $71 \%$ of the students who had the tutorial (populations $\beta$ and $\gamma$, respectively) answer correctly [29]. This is a significant improvement over the pretest results.

\section{CONCLUSION}

The results of this study indicate that understanding the difference between pure superposition states and mixed states is difficult for physics students at all levels. Even students at the beginning of graduate quantum mechanics instruction struggle in a similar way as undergraduates. There is substantial improvement after graduate students have learned the mathematical formalism of density operators, which is used to represent mixed states.

The difference between superposition states and mixed states is critical to understanding how quantum mechanics differs from classical physics. We found that even explicit instruction using clicker questions did not resolve the difficulty that undergraduate students have with this topic. The use of a two-page interactive lecture tutorial from Tutorials in Physics: Quantum Mechanics, however, was much more effective. Students who worked through the lecture tutorial performed much better on post-tests, even when given the following quarter. We also found that they performed better on superposition questions than their peers who did not work through the tutorial.

The combination of results presented leads us to believe that even students at the sophomore level are able to grasp the difference between pure superposition states and mixed states with the use of an interactive lecture tutorial. We believe that attention to these types of conceptual ideas early in quantum mechanics instruction, often before a junior-level QM course, will improve student understanding of more complex ideas.

\section{ACKNOWLEDGMENTS}

The authors would like to thank current and past members of the Physics Education Group at the University of Washington. We would especially like to acknowledge the work of Andrew Crouse in development of preliminary versions of the quantum mechanics tutorials that provided inspiration for this investigation. We would also like the thank UW faculty members Miguel Morales, Daryl Pedigo, Andreas Karch, and John Rehr for welcoming this research into their classrooms. This research was supported in part by the National Science Foundation under Grant No. DUE-1022449. 
[1] Note that superposition is rarely emphasized in this example.

[2] H. Fischler and M. Lichtfeldt, Modern physics and students' conceptions, Int. J. Sci. Educ. 14, 181 (1992).

[3] Research on Teaching and Learning of Quantum Mechanics, Proceedings of the Annual Meeting of the National Association for Research in Science Teaching (1999).

[4] R. V. Olsen, Introducing quantum mechanics in the upper secondary school: A study in Norway, Int. J. Sci. Educ. 24, 565 (2002).

[5] D. Zollman, S. N. Rebello, and K. Hogg, Quantum mechanics for everyone: Hand-on activities integrated with technology, Am. J. Phys. 70, 252 (2002).

[6] S. McKagan, K. K. Perkins, and C. E. Wieman, Reforming a large lecture modern physics course for engineering majors using a PER-based design, AIP Conf. Proc. 883, 34 (2007).

[7] C. Baily and N. Finkelstein, Development of quantum perspectives in modern physics, Phys. Rev. ST Phys. Educ. Res. 5, 010106 (2009).

[8] S. B. McKagan, W. Handley, K. K. Perkins, and C. E. Wieman, A research-based curriculum for teaching the photoelectric effect, Am. J. Phys. 77, 87 (2009).

[9] R. N. Steinberg, G. E. Oberem, and L. C. McDermott, Development of a computer-based tutorial on the photoelectric effect, Am. J. Phys. 64, 1370 (1996).

[10] S. McKagan, K. K. Perkins, M. Dubson, C. Malley, S. Reid, R. LeMaster, and C. E. Wieman, Developing and researching $\mathrm{PhET}$ simulations for teaching quantum mechanics, Am. J. Phys. 76, 406 (2008).

[11] A. Kohnle, D. Cassettari, T. J. Edwards, C. Ferguson, A. D. Gillies, C. A. Hooley, N. Korolkova, J. Llama, and B. D. Sinclair, A new multimedia resource for teaching quantum mechanics concepts, Am. J. Phys. 80, 148 (2012).

[12] A. Kohnle, C. Baily, A. Campbell, N. Korolkova, and M. J. Paetkau, Enhancing student learning of two-level quantum systems with interactive simulations, Am. J. Phys. 83, 560 (2015).

[13] S. McKagan, K. K. Perkins, and C. E. Wieman, Design and validation of the Quantum Mechanics Conceptual Survey, Phys. Rev. ST Phys. Educ. Res. 6, 020121 (2010).

[14] A mixed state is often described as being in either one eigenstate or another, it is just not known which (although the probabilities are often known). This differs from a pure superposition, which is not in one eigenstate or the other, but is in a superposition of both eigenstates.

[15] C. Singh, Transfer of learning in quantum mechanics, AIP Conf. Proc. 790, 23 (2005).
[16] G. Zhu and C. Singh, Improving students' understanding of quantum mechanics via the Stern-Gerlach experiment, Am. J. Phys. 79, 499 (2011).

[17] A. Kohnle, C. Baily, and S. Ruby, Investigating the influence of visualization on student understanding of quantum superposition, in Physics Education Research Conference, Minneapolis, MN, 2014.

[18] D. H. McIntyre, Quantum Mechanics (Pearson Education, Inc., San Francisco, CA, 2012).

[19] M. Kryjevskaia, A. Boudreaux, and D. Heins, Assessing the flexibility of research-based instructional strategies: Implementing tutorials in introductory physics in the lecture environment, Am. J. Phys. 82, 238 (2014).

[20] D. J. Griffiths, Introduction to Quantum Mechanics, 2nd ed. (Pearson Prentice Hall, Upper Saddle River, NJ, 2005).

[21] L. C. McDermott, P.S. Shaffer, and the UW Physics Education Group, Tutorials in Introductory Physics (Prentice Hall College Div., Upper Saddle River, 2001).

[22] http://phet.colorado.edu/en/simulation/stern-gerlach.

[23] L. C. McDermott, P. S. Shaffer, P. R. L. Heron, and the UW Physics Education Group, Tutorials in Physics: Quantum Mechanics, preliminary edition (2013).

[24] Note that a very small number of students did not answer the question, or selected an option that was not one of the three listed. For this reason the numbers for each answer option may not add up to the total number of students.

[25] L. C. McDermott and P. S. Shaffer, Research as a guide for curriculum development: An example from introductory electricity. Part I: Investigation of student understanding, Am. J. Phys. 60, 994 (1992).

[26] P. S. Shaffer and L. C. McDermott, Research as a guide for curriculum development: An example from introductory electricity. Part II: Design of instructional strategies, Am. J. Phys. 60, 1003 (1992).

[27] See Supplemental Material at http://link.aps.org/ supplemental/10.1103/PhysRevSTPER.11.020135 for a copy of the lecture tutorial. It has been adapted from Tutorials in Physics: Quantum Mechanics [23].

[28] There was also a change in the curriculum and textbook used for the sophomore course during this investigation (students in the $\alpha$ category had a different textbook than the $\beta$ and $\gamma$ students). Results from the pre- and post-test data suggest that this change in the course content did not affect student performance on these questions.

[29] Note that this was the third time that students in categories $\beta$ and $\gamma$ had seen this type of question. 